\title{
Feasibility of a quantitative polymerase chain reaction assay for diagnosing pneumococcal pneumonia using oropharyngeal swabs
}

\author{
M. L. van Schaik ${ }^{1,2,4} \cdot$ R. Duijkers ${ }^{1} \cdot$ N. Paternotte ${ }^{1} \cdot$ R. Jansen ${ }^{2} \cdot$ W. Rozemeijer ${ }^{3} \cdot$ W. A. van der Reijden ${ }^{2}$ \\ W. G. Boersma ${ }^{1,5}$
}

Received: 25 June 2018 / Accepted: 6 December 2018 / Published online: 19 December 2018

(c) Springer Nature B.V. 2018

\begin{abstract}
Streptococcus pneumoniae is the most important pathogen causing community-acquired pneumonia (CAP). The current diagnostic microbial standard detects S. pneumoniae in less than $30 \%$ of CAP cases. A quantitative polymerase chain reaction (PCR) targeting autolysin (lytA) is able to increase the rate of detection. The aim of this study is validation of this quantitative PCR in vitro using different available strains and in vivo using clinical samples (oropharyngeal swabs). The PCR autolysin (lytA) was validated by testing the intra- and inter-run variability. Also, the in vitro specificity and sensitivity, including the lower limit of detection was determined. In addition, a pilot-study was performed using samples from patients $(n=28)$ with pneumococcal pneumonia and patients $(\mathrm{n}=28)$ with a pneumonia without detection of $S$. pneumoniae with the current diagnostic microbial standard, but with detection of either a viral and or another bacterial pathogen to validate this test further. The intra- and inter-run variability were relatively low (SD's ranging from 0.08 to 0.96 cycle thresholds). The lower limit of detection turned out to be 1-10 DNA copies/reaction. In-vitro sensitivity and specificity of the tested specimens ( 8 strains carrying $l y t A$ and 6 strains negative for $l y t A$ ) were both $100 \%$. In patients with pneumococcal and non-pneumococcal pneumonia a cut-off value of 6.000 copies $/ \mathrm{mL}$ would lead to a sensitivity of $57.1 \%$ and a specificity of $85.7 \%$. We were able to develop a quantitative PCR targeting lytA with good in-vitro test characteristics.
\end{abstract}

Keywords Streptococcus pneumoniae $\cdot$ Quantitative PCR $\cdot$ Pneumonia $\cdot$ LytA $\cdot$ Community-acquired pneumonia

\section{Introduction}

Streptococcus pneumoniae is the most important pathogen causing community-acquired pneumonia (CAP) [1-3]. The current diagnostic standard, comprised of blood cultures, sputum cultures and the urinary antigen test (UAT), is only able to detect $S$. pneumoniae in less than $30 \%$ of CAP cases $[4,5]$. Furthermore, it takes up to several days to yield a positive result and antibiotic therapy can be narrowed [6]. The UAT is currently the test with the highest sensitivity, ranging from 59 to $87 \%$ and specificity of $94 \%$, increasing the detection of $S$. pneumoniae in patients with CAP from 14.0 to $27.0 \%$ [7]. Detecting S. pneumoniae before or after the start of antibiotic treatment requires a target. Different genes of $S$. pneumoniae have been used in research as a target, including spn9802, pneumolysin (ply), wzg (cpsA), and autolysin $(l y t A)$ by PCR [4, 5, 8-18]. A target gene should be specific for $S$. pneumoniae and be absent in the other non-pneumococcal streptococci such as Streptococcus mitis, Streptococcus oralis and the recently discovered

5 Department of Pulmonary Diseases, Northwest Hospital Group, P.O. Box 501, 1800AM Alkmaar, The Netherlands 
Streptococcus pseudopneumoniae [18]. Remarkably, ply is believed to be less specific for $S$. pneumoniae than lytA $[19,20]$. The ply and $l y t A$ gene have both been found in $S$. mitis strains. The isolates containing these genes were all associated with respiratory disease [21]. One recent study by Albrich et al. [5] showed that quantitative polymerase chain reaction (qPCR) tested on nasopharyngeal (NP) samples targeting the lytA gene in a study population that consisted mainly of HIV-infected adults detected $S$. pneumoniae in $52.5 \%$ of CAP cases. The diagnostic standard (blood culture, sputum Gram stain or culture or UAT) detected S. pneumoniae in only $27.1 \%$ of CAP cases. The combination of target genes has been suggested to improve the reliability of the qPCR. The target gene piaB has been used next to lytA to increase the specificity. A recent study by Simoes et al. [22] used both lytA and piaB to identify $S$. pneumoniae and the addition of piaB led to the discovery of two pneumococcal strains that were not identified by lytA alone. However, the authors mention that piaB is not present in some non-encapsulated pneumococci and some non-typeable pneumococci. An earlier study also combined lytA and piaA for the detection of colonization of the nasopharynx by S. pneumoniae [23]. A strain was considered to be a $S$. pneumoniae species when both genes were present. Four strains did not include the piaA gene, but turned out to be $S$. pneumoniae species.

Using two target genes leads to the difficult situation of interpreting a strain which encompasses one gene, but lacks the other gene. Adding piaB will lead to a lower sensitivity. This means that some patients will be withheld narrowspectrum antibiotics.

LytA encodes for an autolysin that is activated in the presence of antibiotics such as penicillin and detergents such as deoxycholate [24]. It has also been considered to be a virulence factor, which means that it enables $S$. pneumoniae to enter the cells of its host, replicate inside these cells and persist in them [25]. LytA is a stable or conserved gene, which is a favorable target for detection [13]. In 2001 McAvin et al. [13] found that in vitro the lytA gene showed promising results with a sensitivity and specificity of $100 \%$ for $S$. pneumoniae. A more recent study in which clinical samples were used also found a specificity of $100 \%$, but a much lower sensitivity of 53\% [26]. Other research stated that $l y t A$ is not specific enough to differentiate between S. pneumoniae and some strains of S. mitis, S. pseudopneumoniae and S. oralis $[18,27,28]$. However, there are studies that claim that lytA can rarely be found in non-pneumococcal bacteria $[14,15]$.

Streptococcus pneumoniae is a pathogen capable of colonization of the upper respiratory tract [23, 29]. Differentiating between colonization and infection is necessary to detect the patients with true pneumococcal pneumonia. Setting a cut-off value using a qPCR could potentially deal with this problem.
The aim of our study is to set up and validate a quantitative PCR assay targeting the lytA gene for detection of $S$. pneumoniae in adult patients with CAP. First, we validated the assay by examining the quality and reproducibility. Subsequently, the sensitivity and the lower limit of detection (LLOD) of the assay, as well as the specificity of the PCR was tested. After validation, we performed a pilot-study with clinical samples in patients with pneumonia caused by different pathogens.

\section{Materials and methods}

\section{Study outline}

The study was performed in the Regional Laboratory for Public Health Kennemerland in Haarlem between the 1st of September and the 8th of December 2015.

Amplification of a part of the bacterial DNA (the amplicon) using PCR leads to extremely high levels of amplicons after the experiment, in contrast to relatively low levels before the start of the amplification cycles. To check for possible inaccuracy the qPCR assay was compared with one other method of quantification: quantification using universal $16 \mathrm{~S}$ ribosomal RNA primers. The concentration of the sample that was used containing a quality control strain of S. pneumoniae (S. pneumoniae American Type Culture Collection (ATCC) 49619) was calculated with PicoGreen quantification and $16 \mathrm{~S}$ rDNA quantification. This calculated concentration was used to asses a standard curve for a lytA qPCR using primers/probe constructed by Carvalho et al. [26]; forward primer (560 nM): 5'-ACGCAATCTA GCA GATGAAG CA-3'; reverse primer (2800 nM): 5'-TCGTGC GTTT TAATTCCAGC T-3'; probe (700 nM): 5'-FAM-GCC GAAAACG CTTGATACAG GGAG-3'-BHQ1. The standard curve enabled calculation of concentrations from other samples of $S$. pneumoniae and other non-pneumococcal streptococci (provided by the Department of Paediatric Immunology and Infectious Diseases, Wilhelmina's Children Hospital, University Medical Centre Utrecht, Utrecht, The Netherlands). The concentrations of the standard curve were compared to those calculated using $16 \mathrm{~S}$ rDNA quantification performed by aforementioned samples.

\section{Bacterial strains}

Streptococcus pneumoniae ATCC 49619 was used to compare methods and for optimization of the quantitative PCR targeting $l y t A$ as well as assessing a standard curve. A collection of strains was used to test the specificity and sensitivity of the assay. S. pneumoniae strains with known concentrations (OK-2-816; OK-2-1213; OK-2-1214;OK-2-077) and unknown concentrations (serotype 8 ; serotype 14; serotype 
19A; strain 406) were used to test the sensitivity. S. pseudopneumoniae strains (k221; ILI42; OK-3-VE-224; 2120942), as well as a $S$. mitis (S. mitis SK579 (b1019)) and a S. oralis strain (2021933), all lytA negative, were used to test the specificity. The strains were provided by the Department of Paediatric Immunology and Infectious Diseases, Wilhelmina's Children Hospital, University Medical Centre Utrecht, Utrecht, The Netherlands. Concentrations and characteristics are available from the supplementary appendix.

\section{Clinical samples}

Samples were prospectively collected from patients with CAP (REDUCE study; clintrials.gov database NCT01964495). For this present (pilot-)study oropharyngeal (OP) swabs were used. All oropharyngeal swabs were obtained by rolling the swabs on the tonsils and posterior wall of the oropharynx with enough pressure to dislodge cells from the mucosal surface. The oropharyngeal swabs used in this study are eSwab ${ }^{\mathrm{TM}}$ with liquid Amies medium as preservation medium (Copan Italia SpA, Brescia, Italy). Viral pathogens could be identified using a PCR performed on these OP swabs. Of every swab $5 \mu \mathrm{L}$ liquid was added to the primer/probe mix. The patient characteristics are shown in Table 1 .

\section{Isolation of bacterial DNA}

DNA of S. pneumoniae ATCC 49619 was isolated using the Highpure PCR template preparation kit (Roche Diagnostics Nederland BV, Almere, The Netherlands). Bacterial DNA from clinical respiratory samples were obtained by total DNA extraction using a NucliSENS EasyMag total nucleic acid extractor (bioMérieux, Marcy l'Etoile, France). The total nucleic acid component of the sample $(200 \mu \mathrm{L})$ was eluted in a final volume of $40 \mu \mathrm{L}$.

\section{Molecular quantification of bacterial DNA of the positive control (S. pneumoniae ATCC 49619)}

For quantification of a positive control (S. pneumoniae ATCC 49619) we used the Quant-IT PicoGreen dsDNA assay kit (Life Technologies, Bleiswijk, The Netherlands). The fluorescence was measured using a LightCycler® ${ }^{\circledR} 480 \mathrm{II}$ real-time PCR analyser (Roche, Almere, The Netherlands). To convert from the concentration in $n g / \mu \mathrm{L}$ to the number of genome copies per $\mu \mathrm{L}$ the genome size, approximately 2.1 million base pairs was estimated [30].

As a comparison for the Picogreen quantification method, quantification of S. pneumoniae ATCC 49619 was performed using a 16S PCR targeted by universal primers [31].

Table 1 Patient characteristics

\begin{tabular}{|c|c|c|c|c|}
\hline & \multicolumn{4}{|l|}{ Patient characteristics } \\
\hline & \multicolumn{2}{|l|}{ S. pneumoniae $(\mathrm{n}=28)$} & \multicolumn{2}{|l|}{ Other pathogens $(=28)$} \\
\hline Age (year) & $\begin{array}{l}67.38 \pm 16.218 \\
\text { (Range 24-92) }\end{array}$ & & $\begin{array}{l}67.54 \pm 13.226 \\
\text { (Range 44-94) }\end{array}$ & \\
\hline Male & 16 & $57.1 \%$ & 16 & $57.1 \%$ \\
\hline Female & 12 & $42.9 \%$ & 12 & $42.9 \%$ \\
\hline Current smoker & 9 & $32.1 \%$ & 6 & $21.4 \%$ \\
\hline Previous smoker & 13 & $46.4 \%$ & 16 & $57.1 \%$ \\
\hline \multicolumn{5}{|l|}{ CURB-65 } \\
\hline 0 & 5 & $17.9 \%$ & 8 & $28.6 \%$ \\
\hline 1 & 5 & $17.9 \%$ & 11 & $39.3 \%$ \\
\hline 2 & 10 & $35.7 \%$ & 6 & $21.4 \%$ \\
\hline 3 & 8 & $28.6 \%$ & 2 & $7.1 \%$ \\
\hline 4 & 0 & - & 1 & $3.6 \%$ \\
\hline 5 & 0 & - & 0 & - \\
\hline COPD & 11 & $39.3 \%$ & 14 & $50 \%$ \\
\hline Pre-treatment with $\mathrm{AB}$ & 2 & $7.1 \%$ & 7 & $25 \%$ \\
\hline Positive blood culture & 12 & $42.9 \%$ & 3 & $10.7 \%$ \\
\hline Positive sputum culture & 10 & $35.7 \%$ & 11 & $39.3 \%$ \\
\hline Positive urinary antigen test & 13 & $46.4 \%$ & $1^{\mathrm{a}}$ & $3.6 \%$ \\
\hline $\begin{array}{l}\text { Positive pharyngeal swab (viral } \\
\text { pathogens) }\end{array}$ & & & 20 & $71.4 \%$ \\
\hline
\end{tabular}

The patient characteristics of the two groups (infected with $S$. pneumoniae or other viral/bacterial pathogens) of patients admitted with CAP. $A B$ antibiotics. Pharyngeal swabs were only tested for viral pathogens at time of admittance

${ }^{\mathrm{a} P o s i t i v e ~ f o r ~ L e g i o n e l l a ~ p n e u m o p h i l a ~}$ 
The quantitative PCRs were run on LightCycler 1.5 or 2.0 (Roche, Almere, the Netherlands). LightCycler software (Version 4.1) resulted in the calculation of the number of $S$. pneumoniae DNA copies of the positive control.

\section{Quantitative PCR targeting lytA}

The $S$. pneumoniae-quantitative PCR uses primers targeting the lytA gene as described by Carvalho et al. [26]. Roche LightCycler® 480 Probes Master mix was used for all PCR reactions. PCR ran the following program using the LightCycler ${ }^{\circledR} 480$ (Roche): $10 \mathrm{~min}$ at $95{ }^{\circ} \mathrm{C}$, followed by 45 cycles that are comprised of $15 \mathrm{~s}$ at $95^{\circ} \mathrm{C}$ and $1 \mathrm{~min}$ at $60{ }^{\circ} \mathrm{C}$. A standard curve was assessed for the quantitative assay by using the $S$. pneumoniae ATCC 49619 strain. Standard curves (three standard curves, calculated with three different experiments, the average of these curves was used as a final standard curve) were validated using strains with known concentrations (OK-2-816; OK-2-1213; OK-2-1214; OK-2-077), kindly provided by the Department of Paediatric Immunology and Infectious Diseases, Wilhelmina's Children Hospital, University Medical Centre Utrecht, Utrecht, the Netherlands. Inter and intra-run variability were established by determination of triplicate serial dilutions in three independent runs.

\section{Sensitivity of the lytA PCR}

LLOD of the quantitative PCR was determined by multiple serial dilutions of purified DNA from $S$. pneumoniae ATCC 49619 equivalent to from 17,000 to 0.17 copies per $\mu \mathrm{L}$.

\section{Specificity of the lytA PCR}

Specificity of the lytA real-time PCR was defined by testing purified DNA from eight pneumococcal strains. These strains include 4 strains that were non-typeable by culture (OK-2-816; OK-2-1213; OK-2-1214; OK-2-077) as well as serotype 8, serotype 14, serotype 19A and strain 406.

Further determination of the specificity was performed by using 6 strains including 4 strains of $S$. pseudopneumoniae (k221; ILI42; OK3-VE-224; 2120942), 1 strain of S. mitis and 1 strain of $S$. oralis. None of these strains encloses the lytA gene.

\section{Statistical analysis}

The 2 different methods (the concentrations calculated with the lytA qPCR and 16S rDNA quantification) were compared using the Bland-Altman-method [32]. Inter-run variability was calculated by one-way analysis of variance (ANOVA) $[33,34]$. Intra-run variability was also tested by one-way analysis of variance. SPSS statistical software (SPSS version
23 for Windows, Chicago, IL, USA) was used to perform the statistical tests mentioned above. A p-value $<0.05$ was considered as statistically significant.

\section{Results}

\section{Molecular quantification of the positive control ( $S$. pneumoniae ATCC 49619)}

The first step in the validation was molecular quantification of the positive control (S. pneumoniae ATCC 49619). Concentrations calculated with PicoGreen had and $16 \mathrm{~S}$ rDNA experiments resulted in an average concentration of $1.70 \times 10^{6}$ DNA copies $/ \mu \mathrm{L}$.

\section{Validation of the lytA quantitative PCR}

The calculated average concentration of $S$. pneumoniae (ATCC 49619) was used to assess a standard curve. This standard curve had a slope of approximately -3.4 and an efficiency of $95.1 \%$. Four lytA positive strains with known concentrations were used to validate our standard curve.

\section{Intra- and inter-run variability}

To examine the feasibility of the qPCR as a diagnostic tool for CAP, the specificity and sensitivity characteristics are determined. Serial dilutions of $S$. pneumoniae (ATCC 49619) were used to account for intra- and interrun variability. For each step dilution, the standard deviation was calculated. Standard deviations ranged from $0.08 \mathrm{Ct}$-value for the samples with the highest concentration to an average of $0.96 \mathrm{Ct}$-values for the lowest concentration (0.17 DNA copies/ $\mu \mathrm{L}$ or 170 DNA copies/mL). No significant differences were found when testing the inter-run variability with a one-way analysis of variance (ANOVA; p-value ranging from 0.426 to 0.929 ).

\section{In vitro performance of the lytA quantitative PCR}

In total 6 lytA-negative strains were tested. Four strains showed fluorescence, while 2 other strains showed no fluorescence after 45 cycles (supplementary appendix). The 4 lytA-negative strains that did show fluorescence appeared as multiple groups of DNA fragments (shorter than the amplicon of 75 base pairs), meaning they contained an accumulation of waste products. Dilutions of $S$. pneumoniae (ATCC 49619) were used to establish the lower limits of detection (LLOD) of the qPCR targeting lytA. The LLOD ranged from approximately 0.85 (SD $0.96 \mathrm{Ct}$ ) DNA copy to approximately 8.5 (SD 0.36 Ct) DNA copies per well. 
In addition, an attempt was made to identify 8 different lytA-positive $S$. pneumoniae strains. These different strains were all identified with $\mathrm{Ct}$ values ranging from 18 to 25 cycles. Only one strain had a positive result after approximately 36 amplification cycles (Serotype 19A). Testing the lytA-positive and $l y t A$-negative strains resulted in an in-vitro sensitivity and specificity of both $100 \%$. The LLOD was 1-10 copies/reaction.

\section{Pilot-study of in vivo specimens}

OP samples from 28 patients with CAP caused by $S$. pneumoniae and 28 patients with a viral pneumonia or pneumonia with another bacterial pathogen, identified by a positive blood, sputum culture or UAT result were used for this pilot study. Concentrations in the OP swabs tested in the group with $S$. pneumoniae ranged from 0 to 1190 DNA copies/ $\mu \mathrm{L}$; 5 patients had a negative result. Concentrations in the group with other pathogens ranged from 0 to $210 \mathrm{DNA}$ copies $/ \mu \mathrm{L}$; 18 patients had a negative result. The largest Area Under the Curve (AUC) was found for a cut-off value of 6.000 DNA copies/mL (AUC 0.714 with a sensitivity of $57.1 \%$ and a specificity of $85.7 \%$ ) (Figs. 1,2) with a positive predictive value of $80 \%$ and a negative predictive value of $66.7 \%$.

Eleven patients in the $S$. pneumoniae group and 13 patients in the group with other pathogens had a complete composite diagnostic microbial standard (blood culture, sputum culture and UAT). The range of the $S$. pneumoniae group (11 patients) was $0-145$ DNA copies/ $\mu \mathrm{L}$ with one negative result and the concentrations in the group with other pathogens (13 patients) ranged from 0 to 211 DNA copies/ $\mu \mathrm{L}$, with eight negative results. The AUC for this second comparison was also highest with a cut-off value of 6.000 DNA copies/mL (AUC 0.787, with a sensitivity of $72.7 \%$ and a specificity of $84.6 \%$ ). The positive and negative predictive value were $80 \%$ and $78.6 \%$ respectively.

\section{Discussion}

The present study shows that the lytA quantitative PCR is a reliable test in order to detect $S$. pneumoniae in vitro and has the potential to be a reliable test in vivo. In vitro sensitivity and specificity are both $100 \%$. More important the test shows promising results in differentiating between infection and colonization. When tested on a small sample of patients, with a complete diagnostic work-up, a sensitivity and specificity of 72.7 and $84.6 \%$ respectively were reached using a cutoff value of 6.000 copies $/ \mathrm{mL}$. One would expect a low number of DNA copies in patients with colonization without infection. With a sensitive test, which can detect a low number of DNA copies per microliter and makes it possible to set a low cut-off value when this hypothesis is true. The
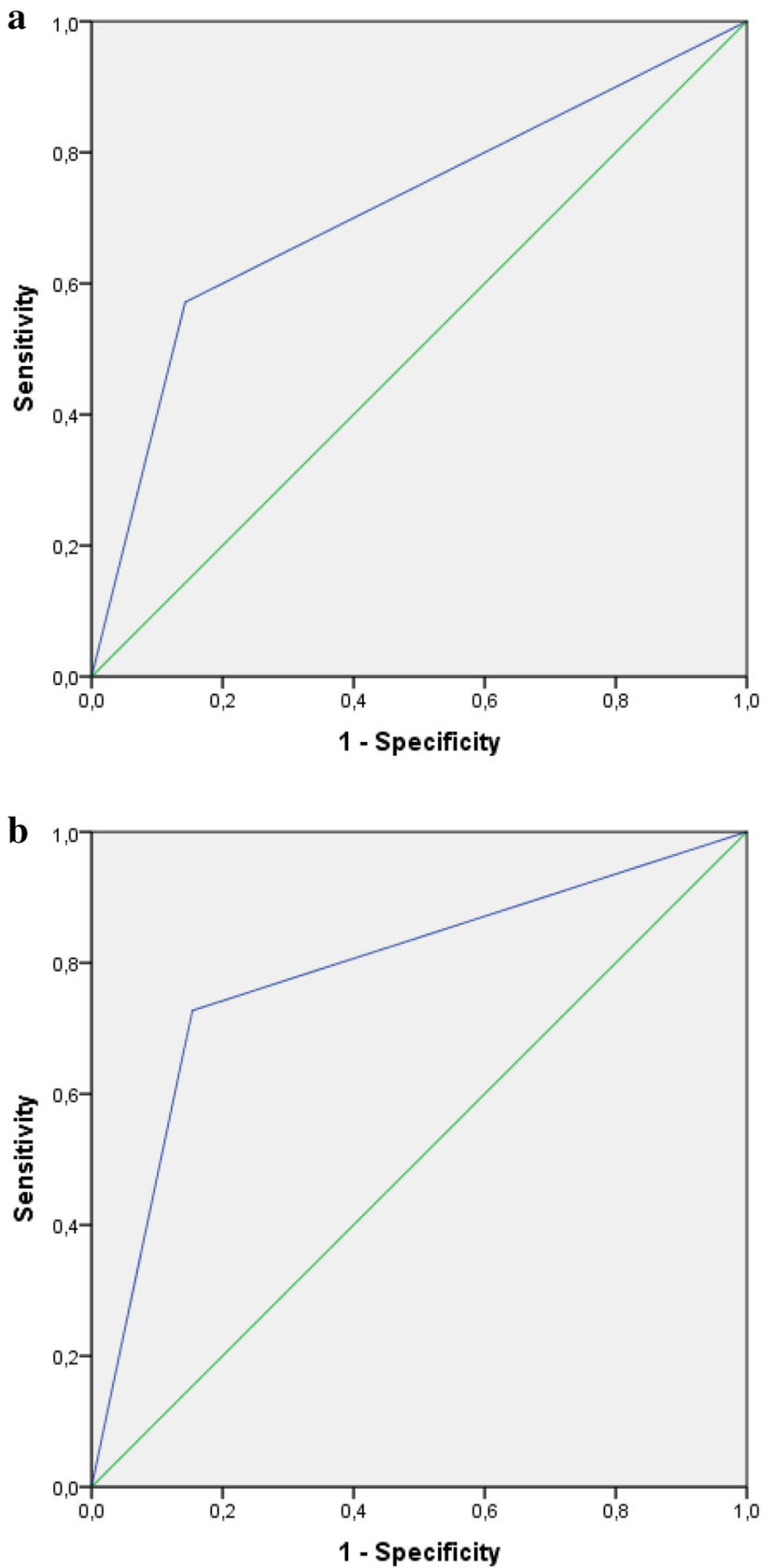

Fig. 1 a ROC-curve with cut-off value 6.000 copies $/ \mathrm{mL}$. Sensitivity is $57.1 \%$ and specificity is $85.7 \%$. AUC is 0.714 . b ROC-curve with a cut-off value of 6.000 copies $/ \mathrm{mL}$. Only samples from patients with a complete composite diagnostic standard (blood culture, sputum culture and urinary antigen tested) performed were used for this curve. Sensitivity is $72.7 \%$ and specificity is $84.6 \%$. AUC is 0.787

in-vitro LLOD turned out to be between approximately 1 and 10 copies $/ \mu \mathrm{L}$, which is similar to the LLOD's found by others varying from $<10$ copies per reaction to 4.3 copies per reaction $[12,15,26]$. This LLOD makes the differentiation between colonization and infection possible. The standard deviations of our standard curve illustrate the reproducibility of our test. 


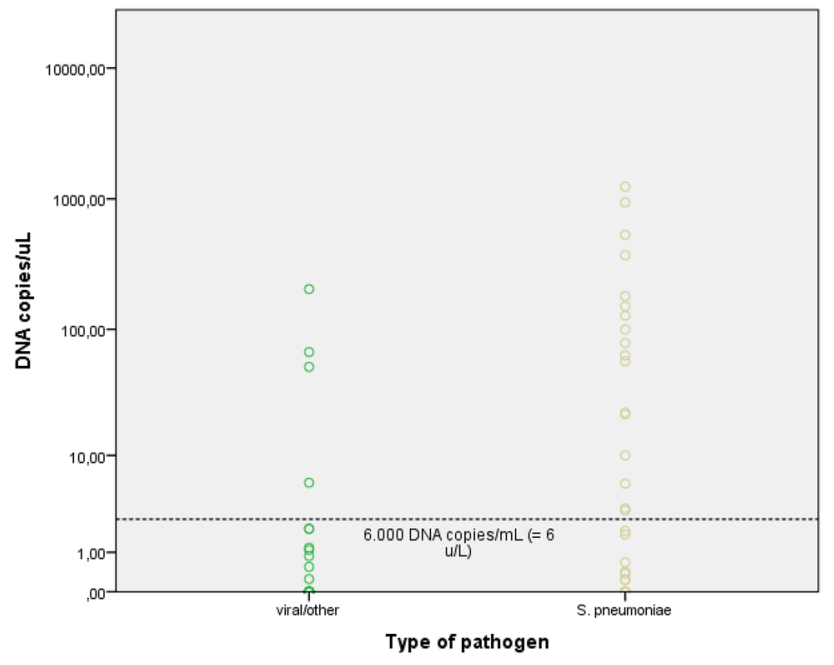

Fig. 2 Pneumococcal load in oropharyngeal swabs. Number of DNA copies/microliter in oropharyngeal swabs in patients with confirmed pneumococcal pneumonia $(n=28)$ or viral/other pathogens $(n=28)$. The dotted line represents the cut-off value of 6.0000 DNA copies/ $\mathrm{mL}$

The specificity and sensitivity are based on a total number of just 14 strains, which is a drawback of our study. In other studies a much larger numbers of pneumococcal strains and controls were tested [13-15]. An in-vitro specificity and sensitivity of $100 \%$ in the first study was found using 70 positive controls and 9 non-pneumococcal streptococci (including 2 S. mitis strains) [13]. This $100 \%$ specificity was confirmed by another study using 23 non-pneumococcal streptococci (including three that closely resemble $S$. pneumoniae; $2 S$. oralis strains and $1 S$. mitis strain) [14]. The largest study tested a total of 257 strains belonging to 37 different species including $30 \mathrm{~S}$. mitis strains, with no false negative results and only one false positive result out of $30 \mathrm{~S}$. mitis strains. This sample was also tested positive by two rapid antigen tests (Wellcogen and Phadebact) [15].

A recent study using the same positive control (ATCC 49619), primers and probe, tested 23 S. pneumoniae strains and 29 negative controls (including six non-pneumococcal species, one being $S$. mitis) [35]. The six negative controls used in the present study are all six closely related to $S$. pneumoniae. Testing these non-pneumococcal strains makes for a valuable contribution to previous trials because they generate signals reported specific to $S$. pneumoniae, in terms of optochin susceptibility, bile solubility, and Quellung testing, the classic methods used to identify pneumococci. These signals make it difficult to discriminate them from pneumococcal strains when performing these tests on blood cultures. However, our PCR was able to discriminate between these strains and S. pneumoniae. The small number of strains tested might overestimate the true specificity. The specificity could be improved by adding a piaB
confirmation-PCR, which can be used for the samples tested positive for lytA.

Our pilot-study consisted of a only small number of patients admitted with either pneumococcal pneumonia or CAP caused by another pathogen. The very small number of samples is a clear limitation of our study. This pilot-study was conducted to perform a preliminary in vivo validation of the qPCR and was not designed as a full clinical trial. A larger population could have resulted in a proper cut-off value, which could be used in further studies or in clinical practice. Although the use of this limited number of OP samples was not intended to define a proper cut-off value, preliminary results are promising: best AUC of 0.714 with a sensitivity of $57.1 \%$ and specificity of $85.7 \%$ with a cutoff value of 6.000 copies $/ \mathrm{mL}$. The AUC was even higher when only using the samples of patients with a complete diagnostic workup; a sensitivity of $72.7 \%$ and a specificity of $84.6 \%$ using a cut-off value of 6.000 copies $/ \mathrm{mL}$ (AUC 0.787). Choosing a different cut-off value to achieve the highest sensitivity may implicate a lower specificity. In clinical practice, in patients who are colonized with $S$. pneumoniae and infected with another bacterial pathogen, the test may be considered as (false) positive and consequently these patients would be treated with narrow-spectrum antibiotic therapy.

Recent research suggested a cut-off value of 8.000 copies $/ \mathrm{mL}$ of the lytA gene when using NP swabs, which led to a sensitivity and specificity of the qPCR of $82.2 \%$ and $92 \%$ respectively [5]. The authors claim that this cut-off value is capable of differentiating between asymptomatic colonization and infection in HIV-infected patients. Another recent study used a much lower cut-off value of $10^{2}$ copies $/ \mathrm{mL}$ [4]. Other researchers, who used the Spn9802 target gene found a similar cut-off value of 4.000-8.000 [10].

A very recent investigation by Blake et al. used a lytA rtPCR on whole blood samples to identify S. pneumoniae in patients with CAP in Togo. The cut-off value was set at a Ctvalue instead of the number of DNA copies $/ \mathrm{mL}$. The cut-off was set on a Ct-value of 35 [36]. The sensitivity of the lyt $A$ rt-PCR was significantly higher than blood culture, $17.1 \%$ versus $12.9 \%$, but has a much lower sensitivity compared to the lytA qPCR we tested on OP swabs. The specificity of the rt-PCR on blood samples was $97.4 \%$. The authors consider this a possible consequence of cross-reactivity with $S$. mitis among other bacteria. This limitation of the lytA PCR has been described in other research as well. One study tested 11 streptococcal isolates that showed conflicting or previously unknown patterns when using optochin susceptibility, bile solubility, lytA PCR and multilocus sequence analysis and discovered that three strains were misidentified with the lytA rt-PCR (one false-negative result and two $S$. pseudopneumoniae strains led to a false-positive result) [22]. In three patients without detection of S. pneumoniae 
using the current diagnostic standard, but with detection of a virus (two coronaviruses and one influenza A virus) concentrations of $S$. pneumoniae above 40.000 copies $/ \mathrm{mL}$ were detected, which limits the specificity of our test in this experiment. A possible explanation for these high concentrations of DNA copies/mL is false-negative results of the current pneumococcal tests. Only one of these patients was pretreated with antibiotics and they all had a favorable outcome with amoxicillin. Given the high DNA concentrations above the cut-off values for colonization and the favorable response to therapy an underlying pneumococcal infection seems very likely.

Previously the usefulness of the qPCR has been questioned in patients who were pre-treated with antibiotics [26, 37]. The total number of patients who have been pre-treated with antibiotics in the present study is rather low (16.1\%) and no reliable conclusion can be made on this topic. We believe this is an important issue not only with qPCR but with any microbiological test, so further studies should address this question [12].

A recent study showed that it is possible to detect 26 respiratory bacteria and viruses with one single test. $85 \%$ of the patients tested had been pre-treated with antibiotic therapy, and still in $78 \%$ of these patients a bacterial pathogen was detected, where only $32 \%$ of cultures were positive [38]. A bacterial pathogen was found in $71.5 \%$ of cases. No blood cultures or urinary antigen tests were included and only mucopurulent sputum was used. S. pneumoniae was detected in $35.6 \%$ of cases.

A possible explanation for the low sensitivity is the cutoff value, which at this point is based on a low number of patients, as mentioned before. When counting all positive results (every patient with a DNA copy number of more than zero) the sensitivity of the qPCR is $82 \%$ ( 23 out of 28 patients). Another possible explanation for the low sensitivity compared to the in-vitro sensitivity is the sample technique or sample site. Some studies use sputum samples which are difficult to obtain, whereas others use NP swabs instead of OP swabs [4, $5,19]$. One of these studies compared trans-nasal and transoral sampling, and concluded that the nasopharynx is the main reservoir for S. pneumoniae [23], but data on the best sampling technique is limited and unclear about which technique is superior. According to the WHO Pneumococcal Carriage Working Group NP samples have a slightly higher sensitivity in detecting colonization with S. pneumoniae in healthy adults and children. A combination of NP and OP samples is recommended for detection of $S$. pneumoniae carriage in adults. There are no current recommendations about molecular diagnostics and detection of $S$. pneumoniae in patients with CAP $[39,40]$. Recent research showed that in healthy adults and adults with influenza-like-symptoms the qPCR targeting lytA and piaA or piaB yielded more positive results than cultures (carriage in healthy adults $20 \%$ using the qPCR vs. $5 \%$ detection using cultures). The detection rate of S. pneumoniae in adults with influenza-like-illness was highest in saliva samples (28\%) followed by OP swabs (11\%), cultures (10\%) and NP swabs (5\%) [41, 42].

Primarily, the test will have to be validated in a larger collection of clinical samples so a distinction between colonization and infection can be made. Another important question is the performance of the qPCR in patients pre-treated with antibiotics. Furthermore, the additional value needs to be determined to see if the qPCR will increase microbiological yield and leads to changes in antibiotic regimes.

In conclusion, we were able validate a quantitative PCR targeting lytA with good in-vitro test characteristics. One to 10 DNA copies per reaction could be detected with an in-vitro sensitivity and specificity of $100 \%$. The results of the in-vivo tests are promising with a sensitivity of $57.1 \%$ and a specificity of $85.7 \%$.

We believe the qPCR targeting lytA could be a rapid and reliable tool for diagnosing pneumococcal CAP, but further research with larger groups is necessary.

Author contributions RD, WvdR, WR, MvS and WB were involved in the conception and design of the study. WvdR, RJ and MvS were involved in method development and molecular testing for the study. MvS, RD, WvdR, NP and WB participated in the drafting of the manuscript. All authors interpreted the data and revised the manuscript critically for important intellectual content. All authors approved the final manuscript.

Funding No sponsors were involved in the study design, sample collection, analysis and interpretation of data, writing or decision to submit the paper for publication. Glaxo Smith Kline and Chiesi both provided an unrestricted grant.

Data availability This study represents a subset of the data collected for the REDUCE study and can be accessed by academic researchers with permission from the principal investigator,WB (w.boersma@NWZ.nl).

\section{Compliance with ethical standards}

Conflict of interest The authors declare that they have no conflicts of interest.

Ethical approval For the REDUCE study ethical approval was obtained through the METC Noord-Holland (Postbus 501, 1800 AM, Alkmaar, The Netherlands) which is now part of the METC of the Amsterdam University Medical Centres.

Informed consent For the REDUCE study informed consent was obtained for taking oropharyngeal swabs.

\section{References}

1. Said MA, Johnson HL, Nonyane BA et al (2013) Estimating the burden of pneumococcal pneumonia among adults: a systematic 
review and meta-analysis of diagnostic techniques. PLoS ONE 8:e60273. https://doi.org/10.1371/journal.pone.0060273

2. Torres A, Blasi F, Peetermans WE et al (2014) The aetiology and antibiotic management of community-acquired pneumonia in adults in Europe: a literature review. Eur J Clin Microbiol Infect Dis 33:1065-1079. https://doi.org/10.1007/s10096-014-2067-1

3. Rozenbaum MH, Pechlivanoglou P, Van Der Werf TS et al (2013) The role of Streptococcus pneumoniae in community-acquired pneumonia among adults in Europe: a meta-analysis. Eur J Clin Microbiol Infect Dis 32:305-316. https://doi.org/10.1007/s 1009 6-012-1778-4

4. Strålin K, Herrmann B, Abdeldaim G et al (2014) Comparison of sputum and nasopharyngeal aspirate samples and of the PCR gene targets lytA and Spn9802 for quantitative PCR for rapid detection of pneumococcal pneumonia. J Clin Microbiol 52:83-89. https:// doi.org/10.1128/JCM.01742-13

5. Albrich WC, Madhi S, Adrian PV et al (2012) Use of a rapid test of pneumococcal colonization density to diagnose pneumococcal pneumonia. Clin Infect Dis 54:601-609. https://doi.org/10.1093/ $\mathrm{cid} / \mathrm{cir} 859$

6. File TM Jr (2003) Community-acquired pneumonia. Lancet 362:1991-2001. https://doi.org/10.1016/S0140-6736(03)15021-0

7. Laijen W, Snijders D, Boersma WG (2016) Pneumococcal urinary antigen test: diagnostic yield and impact on antibiotic treatment. Clin Respir J. https://doi.org/10.1111/crj.12453

8. Johansson N, Kalin M, Giske CG, Hedlund J (2008) Quantitative detection of Streptococcus pneumoniae from sputum samples with real-time quantitative polymerase chain reaction for etiologic diagnosis of community-acquired pneumonia. Diagn Microbiol Infect Dis 60:255-261. https://doi.org/10.1016/j.diagmicrob io.2007.10.011

9. Bjarnason A, Lindh M, Westin J et al (2017) Utility of oropharyngeal real-time PCR for S. pneumoniae and H. influenzae for diagnosis of pneumonia in adults. Eur J Clin Microbiol Infect Dis 36:529-536. https://doi.org/10.1007/s10096-016-2829-z

10. Alpkvist H, Athlin S, Nauclér P et al (2015) Clinical and microbiological factors associated with high nasopharyngeal pneumococcal density in patients with pneumococcal pneumonia. PLoS ONE. https://doi.org/10.1371/journal.pone.0140112

11. Gillespie SH, Ullman C, Smith MD, Emery V (1994) Detection of Streptococcus pneumoniae in sputum samples by PCR. J Clin Microbiol 32:1308-1311

12. Rudolph KM, Parkinson AJ, Black CM, Mayer LW (1993) Evaluation of polymerase chain reaction for diagnosis of pneumococcal pneumonia. J Clin Microbiol 31:2661-2666

13. McAvin JC, Reilly PA, Roudabush RM et al (2001) Sensitive and specific method for rapid identification of Streptococcus pneumoniae using real-time fluorescence PCR. J Clin Microbiol 39:3446-3451

14. Sheppard CL, Harrison TG, Morris R et al (2004) Autolysintargeted LightCycler assay including internal process control for detection of Streptococcus pneumoniae DNA in clinical samples. J Med Microbiol 53:189-195. https://doi.org/10.1099/jmm.0.05460 $-0$

15. Strålin K, Bäckman A, Holmberg H et al (2005) Design of a multiplex PCR for Streptococcus pneumoniae, Haemophilus influenzae, Mycoplasma pneumoniae and Chlamydophila pneumoniae to be used on sputum samples. Apmis 113:99-111. https://doi. org/10.1111/j.1600-0463.2005.apm1130203.x

16. Holter JC, Müller F, Bjørang O et al (2015) Etiology of community-acquired pneumonia and diagnostic yields of microbiological methods: a 3-year prospective study in Norway. BMC Infect Dis 15:64. https://doi.org/10.1186/s12879-015-0803-5

17. Abdeldaim GMK, Strålin K, Olcén P et al (2008) Toward a quantitative DNA-based definition of pneumococcal pneumonia: a comparison of Streptococcus pneumoniae target genes, with special reference to the Spn9802 fragment. Diagn Microbiol Infect Dis 60:143-150. https://doi.org/10.1016/j.diagm icrobio.2007.08.010

18. Arbique JC, Poyart C, Trieu-Cuot P et al (2004) Accuracy of phenotypic and genotypic testing for identification of Streptococcus pneumoniae and description of Streptococcus pseudopneumoniae sp. nov. J Clin Microbiol 42:4686-4696. https://doi.org/10.1128/ JCM.42.10.4686-4696.2004

19. Albrich WC, Madhi S, Adrian PV et al (2014) Genomic load from sputum samples and nasopharyngeal swabs for diagnosis of pneumococcal pneumonia in HIV-infected adults. J Clin Microbiol 52:4224-4229. https://doi.org/10.1128/JCM.01553-14

20. Werno AM, Anderson TP, Murdoch DR (2012) Association between pneumococcal load and disease severity in adults with pneumonia. J Med Microbiol 61:1129-1135. https://doi. org/10.1099/jmm.0.044107-0

21. Whatmore AM, Efstratiou A, Pickerill a P et al (2000) Genetic relationships between clinical isolates of Streptococcus pneumoniae, Streptococcus oralis, and Streptococcus mitis: characterization of "atypical" pneumococci and organisms allied to $S$. mitis harboring $S$. pneumoniae virulence factor-encoding genes. Infect Immun 68:1374-1382. https://doi.org/10.1128/ IAI.68.3.1374-1382.2000

22. Simões AS, Tavares DA, Rolo D et al (2016) LytA-based identification methods can misidentify Streptococcus pneumoniae. Diagn Microbiol Infect Dis 85:141-148. https://doi.org/10.1016/j.diagm icrobio.2016.03.018

23. Trzciński K, Bogaert D, Wyllie A et al (2013) Superiority of trans-oral over trans-nasal sampling in detecting Streptococcus pneumoniae colonization in adults. PLoS ONE 8:11-13. https:// doi.org/10.1371/journal.pone.0060520

24. Paton JC, Andrew PW, Boulnois GJ, Mitchell TJ (1993) Molecular analysis of the pathogenicity of Streptococcus pneumoniae: the role of pneumococcal proteins. Annu Rev Microbiol 47:89-115. https://doi.org/10.1146/annurev.micro.47.1.89

25. Romero P, López R, García E (2004) Characterization of LytAlike N-acetylmuramoyl-L-alanine amidases from two new Streptococcus mitis bacteriophages provides insights into the properties of the major pneumococcal autolysin. J Bacteriol 186:8229-8239. https://doi.org/10.1128/JB.186.24.8229

26. Carvalho MDGS, Tondella ML, McCaustland K et al (2007) Evaluation and improvement of real-time PCR assays targeting lytA, ply, and psaA genes for detection of pneumococcal DNA. J Clin Microbiol 45:2460-2466. https://doi.org/10.1128/JCM.02498-06

27. Suzuki N, Yuyama M, Maeda S et al (2006) Genotypic identification of presumptive Streptococcus pneumoniae by PCR using four genes highly specific for $S$. pneumoniae. J Med Microbiol 55:709-714. https://doi.org/10.1099/jmm.0.46296-0

28. Park HK, Lee HJ, Kim W (2010) Real-time PCR assays for the detection and quantification of Streptococcus pneumoniae. FEMS Microbiol Lett 310:48-53. https://doi.org/10.111 1/j.1574-6968.2010.02044.x

29. Bogaert D, De Groot R, Hermans PWM (2004) Streptococcus pneumoniae colonisation: the key to pneumococcal disease. Lancet Infect Dis 4:144-154. https://doi.org/10.1016/S1473 -3099(04)00938-7

30. Benson D, Clark K, Karsch-Mizrachi I et al (2015) GenBank. Nucleic Acids Res 43:D30-D35. https://doi.org/10.1093/nar/ gku1216

31. Clarridge IIIJE (2004) Impact of $16 \mathrm{~S}$ rRNA gene sequence analysis for identification of bacteria on clinical microbiology and infectious diseases. Clin Microbiol Rev 17:840-862. https://doi. org/10.1128/CMR.17.4.840

32. Bland JM, Altman DG (1986) Statistical methods for assessing agreement between two methods of clinical measurement. Lancet 1:307-310. https://doi.org/10.1016/S0140-6736(86)90837-8 
33. Bland JM, Altman DG (2010) Statistical methods for assessing agreement between two methods of clinical measurement. Int J Nurs Stud 47:931-936. https://doi.org/10.1016/j.ijnur stu.2009.10.001

34. Bland JM, Altman DG (2007) Agreement between methods of measurement with multiple observations per individual. J Biopharm Stat 17:571-582. https://doi.org/10.1080/105434007013294 22

35. Ganaie FA, Govindan V, Kumar KLR (2015) Standardisation and evaluation of a quantitative multiplex real-time PCR assay for the rapid identification of Streptococcus pneumoniae. Pneumonia 25:57-66

36. Blake A, Njanpop-Lafourcade BM, Telles JN et al (2017) Evaluation of chest radiography, lytA real-time PCR, and other routine tests for diagnosis of community-acquired pneumonia and estimation of possible attributable fraction of pneumococcus in northern Togo. Epidemiol Infect 145:583-594. https://doi.org/10.1017/ S0950268816002211

37. Dagan R, Shriker O, Hazan I et al (1998) Prospective study to determine clinical relevance of detection of pneumococcal DNA in sera of children by PCR. J Clin Microbiol 36:669-673

38. Gadsby NJ, Russell CD, Mchugh MP et al (2016) Comprehensive molecular testing for respiratory pathogens in communityacquired pneumonia. Clin Infect Dis 62:817-823. https://doi. org/10.1093/cid/civ1214
39. Satzke C, Turner P, Virolainen-Julkunen A et al (2013) Standard method for detecting upper respiratory carriage of Streptococcus pneumoniae: updated recommendations from the World Health Organization Pneumococcal Carriage Working Group. Vaccine 32:165-179. https://doi.org/10.1016/j.vaccine.2013.08.062

40. Watt JP, O'Brien KL, Katz S et al (2004) Nasopharyngeal versus oropharyngeal sampling for detection of pneumococcal carriage in adults. J Clin Microbiol 42:4974-4976. https://doi.org/10.1128/ JCM.42.11.4974-4976.2004

41. Krone CL, Wyllie AL, van Beek J et al (2015) Carriage of Streptococcus pneumoniae in aged adults with influenza-likeillness. PLoS ONE 10:e0119875. https://doi.org/10.1371/journ al.pone. 0119875

42. Wyllie AL, Rümke LW, Arp K et al (2016) Molecular surveillance on Streptococcus pneumoniae carriage in non-elderly adults; little evidence for pneumococcal circulation independent from the reservoir in children. Sci Rep 6:1-9. https://doi.org/10.1038/srep3 4888

Publisher's Note Springer Nature remains neutral with regard to jurisdictional claims in published maps and institutional affiliations. 